\title{
Large-scale climate variability in Antarctica and the Southern Ocean and links to extra-polar climate
}

\author{
Sarah Gille', J. Jones ${ }^{2}$ and H. Goosse \\ La Jolla, USA, 24-26 March 2015
}

Given the short length of the observational record at the high latitudes of the Southern Hemisphere, modern instrumental data are not sufficient to describe the regional state and variability of the climate system. Data rescue of early observations, instrumental data, and proxy records are needed even more here than in other regions. Furthermore, the links between atmosphere, ocean, and sea ice are strong in the southern polar region. The objective of this workshop was to study changes in largescale patterns of Antarctic climate variability over the last decades to centuries, as well as extrapolar-polar connections, by combining proxy records, historical data, and instrumental records that cover the atmospheric, land, and oceanic domains, with model results.

The workshop was attended by an international group of 30 scientists, including early-career scientists. It brought together expertise from the atmosphere, ocean, and sea ice communities, along with expertise in satellite, observational, historical and paleoclimate data, and modeling. It was jointly supported by PAGES and the World Climate Research Programme (WCRP) as part of the WCRP Polar Climate Predictability Initiative (PCPI). The PCPI has a focus on polar regions and their role in the global climate system, and aims to improve predictability of the climate system on all timescales by improving understanding of the underlying physical mechanisms and their representation in climate models.

After reviewing our current knowledge, participants focused on discussing the difficulty in determining the magnitude and causes of decadal-scale variability in climate system changes in the Southern Ocean and Antarctic region. For example, for data types such as those indicated in Figure 1, we considered how to determine whether trends in recent decades are unprecedented or within the range of natural variability. These difficulties are both due to our incomplete understanding of the dynamics of the system and the lack of data. In particular, modern instrumental records in the ocean typically represent isolated point observations, not extended time series, and these records extend back only a few years. Furthermore, paleoclimatic records of sufficiently high resolution are scarce. This severely limits our ability to estimate the natural multi-decadal variability.
Participants found the workshop to be stimulating and would definitely encourage similar jointly supported PAGES and WCRP activities in the future. Workshops such as these provide a rare opportunity for communities that do not normally meet (paleoclimate, instrumental, modeling) to share their expertise covering all aspects of the problem (atmosphere, ocean, sea ice) and to focus on specific issues. Data scarcity was recognized as a crucial limitation for Southern polar climate studies, and the importance of new work that needs to be done to obtain data for oceanic areas in the Southern Hemisphere was stressed, namely by developing new proxies using cold corals and mollusks, and also by imaging, digitizing, and processing meteorological observations from ships' logbooks. The need for better estimates of the uncertainties in current reanalysis products and for a coupled reanalyses dataset focused on high southern latitudes were also underlined.

The meeting achieved its aims of taking stock of current knowledge and determin ing key questions. Participants developed concrete plans for a publication summarizing the meeting findings. The meeting also sowed the seeds for a number of future collaborations, and we have been working to collect information about these emerging collaborative efforts, which we would be happy to share.

\section{AFFILIATIONS}

'Scripps Institution of Oceanography, University of California San Diego, USA

2Department of Geography, University of Sheffield, UK ${ }^{3}$ ELIC/TECLIM Unversité catholique de Louvain, Belgium

CONTACT

Sarah Gille: sgille@ucsd.edu

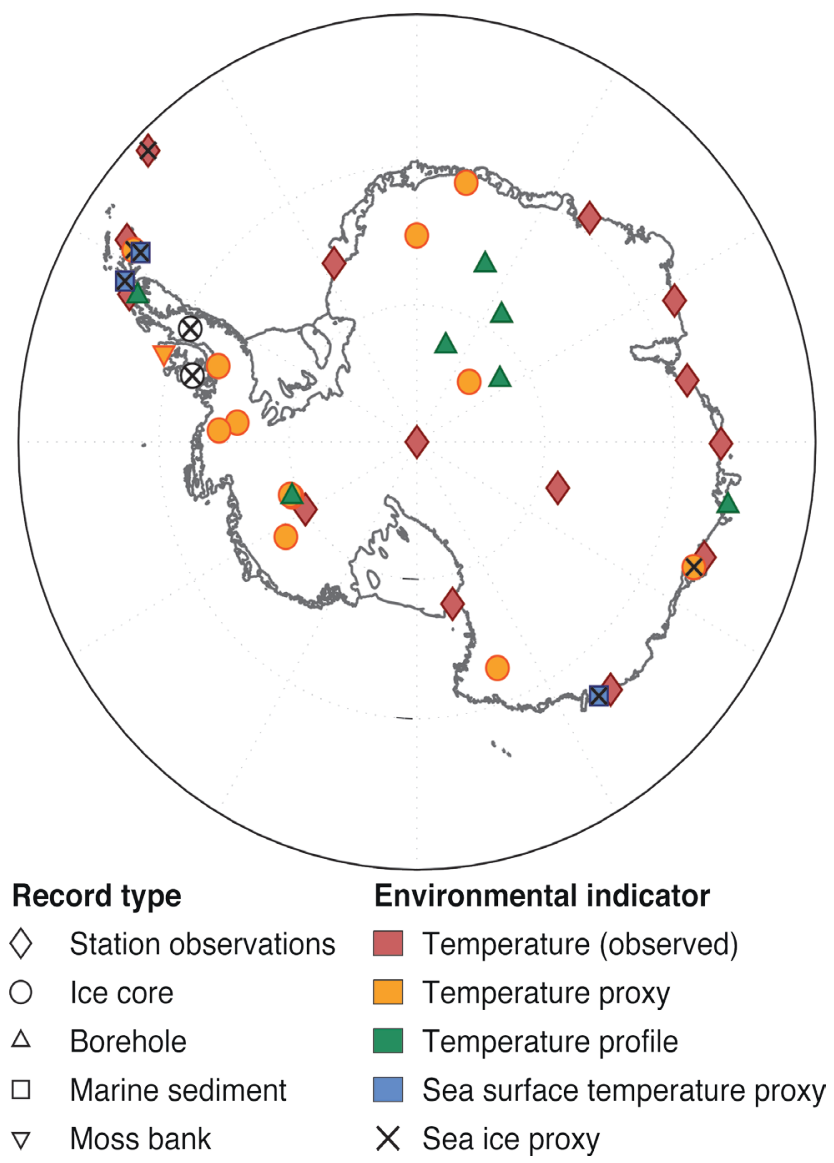

Figure 1: Location and type of climate and environmental information covering the last 200 years in Antarctica (Figure from Nerilie Abram). 